\title{
Remote monitoring of vigilance behavior in large herbivores using acceleration data
}

Max Kröschel ${ }^{1,2^{*}} \mathbb{0}$, Björn Reineking ${ }^{3,4}$, Felicitas Werwie ${ }^{2}$, Felix Wildi ${ }^{1}$ and Ilse Storch ${ }^{1}$

\begin{abstract}
Background: Biotelemetry offers an increasing set of tools to monitor animals. Acceleration sensors in particular can provide remote observations of animal behavior at high temporal resolution. While recent studies have demonstrated the capability of this technique for a wide range of species and behaviors, a coherent methodology is still missing (1) for behavior monitoring of large herbivores that are usually tagged with neck collars and frequently switch between diverse behaviors and (2) for monitoring of vigilance behavior. Here, we present an approach that aims at remotely monitoring different types of large herbivore behavior including vigilance with acceleration data.

Methods: We pioneered this approach with field observations of eight collared roe deer (Capreolus capreolus). First, we trained a classification model for distinguishing seven structural behavior categories: lying, standing, browsing, walking, trotting, galloping and 'others'. Second, we developed a model that predicted the internal states, active and resting, based on the predicted sequence of structural behaviors and expert-based rules. Further, we applied both models to automatically monitor vigilance behavior and compared model predictions with expert judgment of vigilance behavior. To exemplify the practical application of this approach, we predicted behavior, internal state and vigilance continuously for a collared roe deer.
\end{abstract}

Results: The structural behaviors were predicted with high accuracy (overall cross-validated accuracy $71 \%$ ). Only behaviors that are similar in terms of posture and dynamic body movements were prone to misclassification. Active and resting states showed clear distinction and could be utilized as behavioral context for the detection of vigilance behavior. Here, model predictions were characterized by excellent consistency with expert judgment of vigilance behavior (mean accuracy 96\%).

Conclusion: In this study, we demonstrated the strong potential and practical applicability of acceleration data for continuous, high-resolution behavior monitoring of large herbivores and showed that vigilance behavior is well detectable. In particular, when combined with spatial data, automated behavior recognition will enrich many fields in behavioral ecology by providing extensive access to behaviors of animals in the wild.

Keywords: Accelerometry, Animal behavior, Internal state, Behavior monitoring, Roe deer, Capreolus capreolus

\section{Background}

Solid data about animal behavior are essential for many fields of science and management. In the past, collecting these kinds of data was associated with time-consuming field observations and was seldom possible with elusive,

\footnotetext{
*Correspondence: max.kroeschel@wildlife.uni-freiburg.de

${ }^{1}$ Chair of Wildlife Ecology and Wildlife Management, University

of Freiburg, Tennenbacherstr. 4, 79106 Freiburg, Germany

Full list of author information is available at the end of the article
}

nocturnal, wide-ranging or aquatic species. Advances in animal-borne sensors now allow for an automated remote monitoring of animal behavior. Starting in the 1990s, the use of GPS telemetry stimulated substantial progress in animal ecology [1]. By analyzing animal trajectories, researchers also started to gain information about animal behavior that was driving the observed movement pattern [2-4].

Currently, sensors that measure acceleration in different dimensions and with high frequency are about to 
provoke a new boost in ecology [5]. Unlike GPS sensors, which only allow for detecting behaviors that produce distinct movement patterns, acceleration sensors allow for monitoring of a wide variety of behaviors by providing information about an animal's posture and motion. This is technically feasible because acceleration sensors measure two types of acceleration: static body acceleration and dynamic body acceleration [6]. Static body acceleration originates from the earth's gravitational field and is used to calculate the posture of the sensor (see detailed description in [6]). Dynamic body acceleration originates from all motions of the body part to which the sensor is attached. To obtain valid information about animal behavior from acceleration data, it is necessary to identify characteristic signatures inside the data that are correlated with the target behaviors. The methodological steps for automating this task have been extensively described $[7,8]$ and include data collection, data processing, modeling and model application. Acceleration is either measured continuously or over discrete time segments of fixed width, which are called 'bursts.' After data collection, the raw acceleration data are processed into meaningful predictor variables that reflect features of static or dynamic body acceleration. When acceleration is sampled continuously, the signal is first divided into segments of fixed width (e.g., $5 \mathrm{~s}$ ) from which the predictor variables are then calculated (i.e., the predictor variables summarize the acceleration data of the segment, and each segment with its set of predictor variables constitutes an observation). When acceleration is already sampled in bursts, the original bursts or subsegments are used for parameterization. Modeling is usually based on supervised classification models that are trained with a ground-truthed dataset [7-9]. In this case, the behavior of tagged animals must be observed in the field, which allows for assigning a behavioral category to each segment of the training dataset. Alternatively, unsupervised clustering combined with a subsequent expert-based interpretation of the identified clusters can be used [10]. The resulting model can then be used to automatically detect behavior. Automatic classification of animal behavior has been successfully applied in many mammals, birds, fish and reptiles (see recent summary in [5]). The current state of the art of parameterizing the acceleration signal in segments of fixed width, however, has two major disadvantages. First, segments during which the animal displayed several behaviors will deteriorate the performance of the resulting model $[7,11]$. This is particularly problematic in species like roe deer which frequently change behavior. Second, the segment width is ideally adjusted to the acceleration signature of the target behavior. When several behaviors are targeted (e.g., browsing and standing), segments of fixed width are unable to account for behavior-specific signatures of different length.

Automatic monitoring of vigilance behavior with acceleration data is a particular challenge that, to the best of our knowledge, has not been addressed so far. Hereafter, we refer to structural behavior categories as those that are characterized by the structure of the behavior, i.e., by a specific motion and posture (e.g., 'standing with head up') [12]. Further, we refer to an animal's internal state as the operationalized physiological and psychological state of an animal [13] that drives the animal to perform specific structural behaviors to fulfill one or more needs. Many species that are subject to predation perform structural behaviors that fulfill safety needs by reducing the risk of being killed [14, 15]. Large herbivores for example frequently display the structural behavior 'standing with head up' which is commonly interpreted as vigilance behavior by which animals aim for monitoring their surroundings for approaching predators and, thereby, reduce the probability of an attack [14-16]. Behavioral ecologists are most often not per se interested in the display of vigilance behavior but want to measure the internal state of vigilance [16] (e.g., different levels of alertness) which is also referred to as perceived predation risk [14, 17]. The internal state, however, is not directly observable but can be assessed by measuring the outward signs (e.g., the proportion of time or the frequency an animal displays vigilance behavior [16]). During periods when an animal is considered as being in state active or in state foraging, displays of the behavior 'standing with head up' are considered good markers for measuring vigilance/perceived predation risk because herbivores have to switch between different structural behaviors (e.g., feeding with head down standing with head up; Fig. 1) to fulfill safety and other needs (e.g., nutritional needs). Thus, they have to balance conflicting needs in their behavioral decisionmaking process and should invest more time in vigilance when perceived risk increases [14, 17]. While resting (prevailing needs are safety, regeneration and processing of food), an animal might display the structural behavior 'standing with head up' as well (Fig. 1). Here, it might also be dedicated to fulfill safety needs but cannot be used as indicator of vigilance because the animal is not forced to trade between at least two different structural behaviors. Measuring vigilance, therefore, requires (1) monitoring of the structural behavior 'standing with head up' and (2) evaluating the current internal state (i.e., not resting). In field observations that focus on vigilance behavior, the evaluation of an animal's current state is usually handled by observer judgment if the focal animal is active or foraging [18-20]. When automated remote monitoring of vigilance behavior with acceleration data is targeted, 


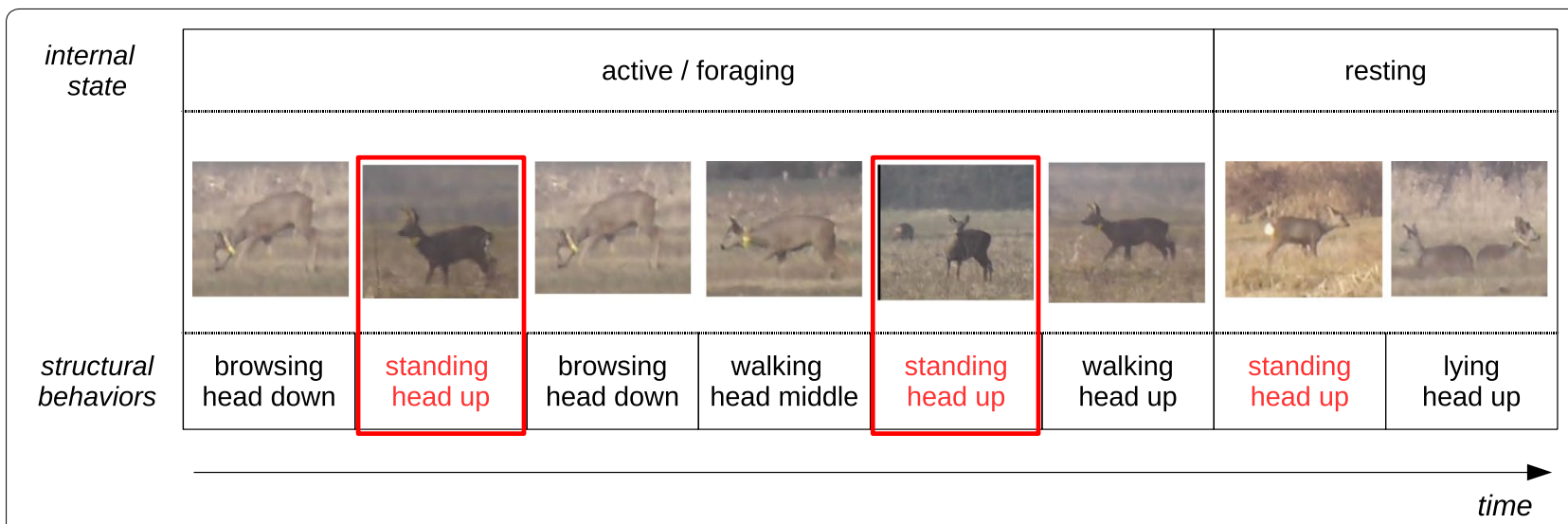

Fig. 1 Illustration of structural behaviors (bottom line and pictures), internal states (top line) and vigilance behavior (red rectangles). Large herbivores switch between different structural behaviors to satisfy prevailing needs (e.g., nutritional and safety needs) when being in state active. Here, a display of the structural behavior 'standing with head up' is referred to as vigilance behavior and can be utilized as index for perceived predation risk. 'Standing with head up' is also displayed during resting states (e.g., when animals stand in the sun for longer periods of time). Here, it can not be used as indicator of vigilance, because the animal is not forced to trade different structural behaviors to fulfill prevailing needs

procedures that predict the internal state of an animal have to be implemented in the modeling framework.

The primary aim of this study is to develop an approach for an automated detection of vigilance behavior in large herbivores, based on acceleration data. We apply the approach to detect vigilance behavior in roe deer (Capreolus capreolus). In the first step, we develop and evaluate a classification model that aims at classifying behavior of roe deer into structural behavior categories, based on video-documented field observations of collared animals. In the second step, we develop a model that synthesizes the sequence of predicted structural behaviors into the internal states 'active' and 'resting. Finally, we apply both models to detect vigilance behavior and compare model predictions with expert judgment of vigilance behavior. We exemplify the application of this approach by predicting behavior, state and vigilance for a collared roe deer.

\section{Methods}

\section{Study area}

Fieldwork for this study was conducted in southwestern Germany (state of Baden-Wuerttemberg) at two study sites (Rhine valley: 48.67, 8.00; Hegau: 47.88, 8.73). Both sites were characterized by woody patches that were surrounded by arable fields and meadows. During the winter months, the vegetation height in all meadows and most fields was very low, allowing for good visibility of several $100 \mathrm{~m}$. A dense network of paved and gravel roads was present at both sites.

\section{Capture and handling of animals}

From 2011 to 2013, we captured roe deer in box-traps and drive-nets. All deer that weighed more than $15 \mathrm{~kg}$ and showed good health were equipped with a neck collar. All capture, tagging and monitoring protocols were approved by the animal welfare and hunting administration of Baden-Wuerttemberg. In total, we collared 47 animals (Rhine valley 35, Hegau 12). The collars were made by e-obs $\mathrm{GmbH}$ (Munich, Germany), weighed $370 \mathrm{~g}$ and, thus, reached maximally $2.5 \%$ of the body mass of tagged deer. The collar itself was made out of leather and hosted a GPS sensor, an acceleration sensor and a UHF transmitter in a case on top and two batteries (D-cells) on each side (Additional file 1: Fig. S1). The weight and the low center of gravity of the batteries prevented the collar from turning around the neck and positioned the GPS/acceleration unit at a dorsal position. The acceleration sensor measured acceleration in three perpendicular axes. Based on the dorsal position, the axis measured acceleration in forward-backward horizontal motion $(x$ axis, denoted as surge), left-right horizontal motion ( $y$ axis, denoted as sway) and up-down vertical motion $(z$ axis, denoted as heave). We measured acceleration every minute at a sampling rate per axis of $10.54 \mathrm{~Hz}$ in bursts of $9.1 \mathrm{~s}$ in length, whereby each axis was sampled alternatingly (xyzxyzxyz...). GPS-positions were taken based on an accelerometer-informed schedule [21]. Once the variance of five consecutive acceleration bursts of the $z$ axis was below a threshold of 1000 (inactive animal, collar settings ACC_L_THR: 1000), the collar collected a GPSposition every $2 \mathrm{~h}$, above this threshold every $15 \mathrm{~min}$ (active animal).

\section{Behavioral observations}

All behavioral observations were made in the winter months of 2011 and 2012 by two observers. In the field, 
the observer first located the collared animals within range via UHF triangulation and afterward placed himself in a favorable position inside a car and waited for approaching animals. To increase the amount of acceleration data during field observations, some collars were rescheduled to measure acceleration bursts every $15 \mathrm{~s}$ for the observation period. Almost all observations were done on meadows due to the low visibility inside the forest. We documented the observed behavior with a video camera (Panasonic Lumix DMC-TZ 10, Panasonic HC-V500). Before or after each observation, a digital radio-controlled clock, which automatically synchronized to the German DCF77 time signal, was filmed to facilitate easy synchronization of acceleration data and video segments.

\section{Data processing}

In the laboratory, we first synchronized the acceleration signals with the video recordings and allocated a structural behavior category to each acceleration measurement. For this purpose, we used the programs Windows Movie Maker 5.1 in combination with $\mathrm{R}$ [28] and the program Observer ${ }^{\circledR}$ XT 10 by Noldus Information Technology. We described behavior based on the structural behavior categories: lying, standing, browsing, walking, trotting, galloping and 'others' (Table 1 with detailed description).

Additionally, we classified the posture of the animal's neck in head down, head middle, head up and head moving (Table 2 with detailed description), because roe deer

\section{Table 1 Ethogram of animal structural behavior}

\begin{tabular}{|c|c|c|}
\hline Behavior & Label & Description of behavior \\
\hline Lying & I & Lying on the ground without body movements \\
\hline Standing & s & Standing without body movements \\
\hline Browsing & $\mathrm{b}$ & $\begin{array}{l}\text { Ingestion of food alternated with single steps } \\
\text { between food sources }\end{array}$ \\
\hline Walking & w & Walking \\
\hline Trotting & $\mathrm{t}$ & Trotting \\
\hline Galloping & g & Galloping \\
\hline Others & 0 & $\begin{array}{l}\text { Shaking, scratching with antler, scratching with hoof, } \\
\text { grooming }\end{array}$ \\
\hline
\end{tabular}

Table 2 Ethogram of animal posture

\begin{tabular}{lll}
\hline Posture & Label & Description of posture \\
\hline Head up & $u$ & Head is kept above shoulder height \\
Head middle & $m$ & Head is kept at shoulder height \\
Head down & d & Head is kept below shoulder height \\
Head moving & mo & $\begin{array}{l}\text { Head is moving from one posture to another } \\
\text { (e.g., from down to up) }\end{array}$ \\
\hline
\end{tabular}

can display all behaviors with different neck postures. All behavior and posture categories were exhaustive and exclusive.

The acceleration data were sampled in units of millivolts. We transformed the raw acceleration data in units of $\mathrm{g}\left(1 \mathrm{~g}=9.81 \mathrm{~m} / \mathrm{s}^{2}\right)$ with a linear transformation following the user manual [22]. As every sensor is a little different, it is recommended to calibrate each collar by successively aligning every axis perpendicular toward the earth's gravitational field. This allows for calculating sensor-specific offset and slope (see detailed description in [22]). We were not able to do the calibration for half of the collars, because we did not calibrate them before collaring the animals and afterward did not get all collars back from the field. Instead, we used the average calibration values of 30 calibrated collars as constants for the linear transformation. Calibration revealed small differences in the collar-specific offsets which influenced measurements of static body acceleration. To prevent potential bias in the classification model, we calculated a collar-specific correction value (see detailed description in Additional file 2: Fig. S2) that was used to calculate a centered static body acceleration of the $x$ axis (sba_x_c, Table 3). We calculated static body acceleration, dynamic body acceleration, mean dynamic body acceleration, mean difference and variance between adjacent data points, variance of static body acceleration, variance and maximum of dynamic body acceleration, dominant power spectrum and the frequency of the dominant power spectrum (see Table 3 with detailed description). Most predictor variables were already used in former studies [7, 23, 24].

Instead of assigning predictor variables to segments of fixed width, we calculated those for each data point based on moving windows with variable width (Fig. 2). Static body acceleration was calculated with a moving average using a window width of seven data points $(0.66 \mathrm{~s})$. This window width showed fine smoothing when animals changed posture [25]. All other predictor variables were calculated with window widths of 10 data points $(\sim 1 \mathrm{~s}$, labeled as w1), 22 data points ( $2 \mathrm{~s}$, labeled as w2), 45 data points $(\sim 4 \mathrm{~s}$, labeled as w5) and 96 data points $(\sim 9 \mathrm{~s}$ labeled as w9).

\section{Modeling}

The modeling approach consisted of three modules. In the first module, we developed and evaluated a classification model that classified the observed acceleration signal into structural behavior categories. We expanded this module with another classification model that targeted the posture of an animal's neck. In the second module, we developed a model to synthesize the predicted sequence of structural behaviors into the internal states active and 
Table 3 Description of predictor variables

\begin{tabular}{|c|c|c|}
\hline Name & Formula & Description \\
\hline sba & $s b a_{i}=\frac{1}{7} * \sum_{j=i-3}^{i+3} a c c_{j}$ & Static body acceleration \\
\hline sba_x_c & sba_x_c $c_{i}=s b a \_x_{i}-$ correction_value tag & Corrected static body acceleration of $x$ axis \\
\hline $\mathrm{dba}$ & $d b a_{i}=\left|a c c_{i}-s b a_{i}\right|$ & Dynamic body acceleration \\
\hline mdba & $m d b a_{i}=\frac{1}{w} \sum_{j=i-\frac{w}{2}}^{i+\frac{w}{2}} d b a_{i}$ & Mean dynamic body acceleration \\
\hline mdba_xyz & $m d b a_{-} x y z_{i}=\sum_{j=i-\frac{w}{2}}^{i+\frac{w}{2}}\left(d b a_{-} x_{j}+d b a_{-} y_{j}+d b a_{-} z_{j}\right)$ & Overall mean dynamic body acceleration of the $x-, y$ - and $z$-axis \\
\hline meandl & meand $_{i}=\frac{1}{w} \sum_{j=i-\frac{w}{2}}^{i+\frac{w}{2}}\left|a c c_{j+1}-a c c_{j}\right|$ & Mean absolute difference between adjacent data points \\
\hline vardl & $\operatorname{vardl}_{i}=\frac{1}{w-1} \sum_{j=i-\frac{w}{2}}^{i+\frac{w}{2}}\left(\left|a c c_{j+1}-a c c_{j}\right|-\text { meandl }_{i}\right)^{2}$ & Variance of the absolute difference between adjacent data points \\
\hline varsba & $\operatorname{varsba}_{i} \frac{1}{w-1} \sum_{j=i-\frac{w}{2}}^{i+\frac{w}{2}}\left(s b a_{j}-\overline{s b a}\right)^{2}$ & Variance of the static body acceleration \\
\hline vardba & $\operatorname{vardb}_{i}=\frac{1}{w-1} \sum_{j=i-\frac{w}{2}}^{i+\frac{w}{2}}\left(d b a_{j}-\overline{d b a}\right)^{2}$ & Variance of the dynamic body acceleration \\
\hline maxdba & $\max d b a_{i}=M A X_{j=i-\frac{w}{2}}^{i+\frac{w}{2}}\left(d b a_{j}\right)$ & Maximum of the dynamic body acceleration \\
\hline dps & See R-Script (Additional file 8). & Dominant power spectrum \\
\hline fdps & See R-Script (Additional file 8). & Frequency of the dominant power spectrum \\
\hline
\end{tabular}

( $i=$ data point within the burst, $w=$ window width). Each predictor variable is calculated for each acceleration axis (except odba_xyz) and for different window widths (except sba). These are indicated by specific suffixes (e.g., meandl_x_w2 stands for the meandl that is calculated over the acceleration data from the $x$ axis with a window width of $2 \mathrm{~s}$ )

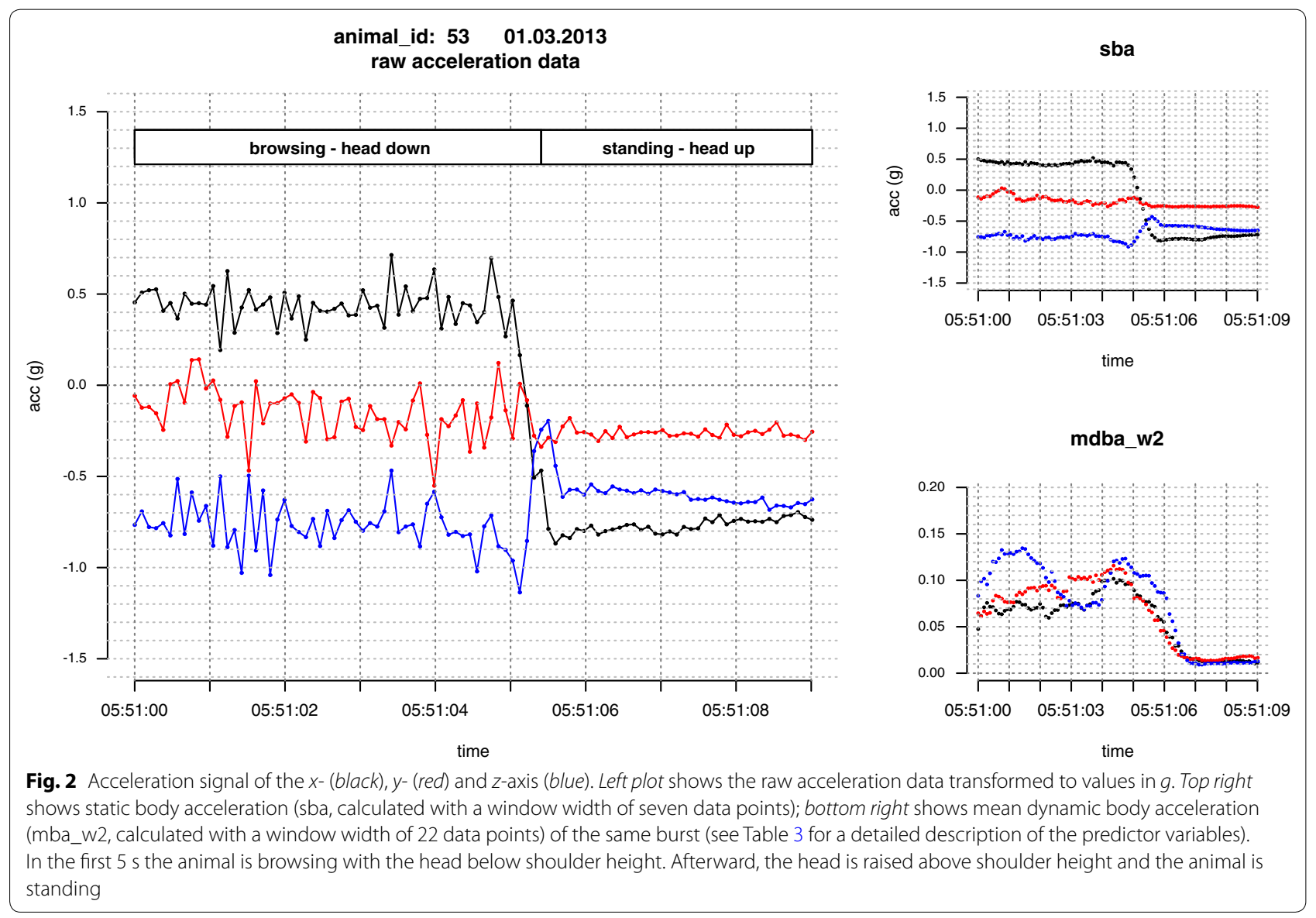


resting. In the third module, we combined the previous modules to predict vigilance behavior.

Previous studies that tested different classification techniques to classify behavior from acceleration data did not find substantial differences in terms of model performance $[8,26]$. Therefore, we employed classification trees (CART algorithm, $\mathrm{R}$ package rpart $[27,28]$ ) which are relatively fast-running and straightforward to interpret. The amount of behavior-tagged acceleration data differed between the observed animals (Additional file 3: Fig. S3). In case of differences in the individual acceleration signatures, this would have caused biased splitting rules toward animals with more data. Hence, we homogenized the dataset and randomly selected a subset of 1500 data points per behavior category and animal. We evaluated the minimum amount of data points per subset and observed a stabilization of the splitting rules after 1000 data points. For some animals, we had less than 1500 data points for the behavior categories trotting, galloping and 'others'. Here, we used all available data points. The resulting dataset was split into $2 / 3$ training and $1 / 3$ validation datasets. We were not particularly interested in the behavior category 'others' and reduced the weight (model parameter 'weight') for these data points until 'others' behavior was classified into a single leaf. Preliminary testing showed that model calibration based on random tenfold cross-validation led to substantial overfitting of the models. We therefore used cross-validation based on the individual animals to determine the optimal tree size. Here, the model is trained successively for $x-1$ animals and tested for the out-of-bag animal. We let the classification tree grow to full size and then pruned it to the smallest tree whose cross-validation error is less than the minimum cross-validation error plus one standard deviation ('1-SE rule', [27]). We evaluated model performance by predicting the pruned classification model to the validation dataset and calculated model accuracy

accuracy

$$
=\frac{\text { number of true positives }+ \text { number of true negatives }}{\text { total number of datapoints }},
$$

sensitivity

$$
\begin{aligned}
\text { sensitivity } & =\frac{\text { number of true positives }}{\text { number of true positives }+ \text { number of false negatives }} \\
& =\frac{\text { number of true positives }}{\text { number of all positives }}
\end{aligned}
$$

and positive predictive value

$$
\begin{aligned}
\text { ppp } & =\frac{\text { number of true positives }}{\text { number of true positives }+ \text { number of false positives }} \\
& =\frac{\text { number of true positives }}{\text { number of positive calls }}
\end{aligned}
$$

for each animal separately. Furthermore, we developed and evaluated a classification model that predicted the posture of the animal's neck (posture model). For this model, we selected parameters that reflect neck posture (sba_x, sba_x_c and sba_z) as predictor variables and excluded data points when the head was moving (posture 'head moving'). We applied the same model procedure as for the behavior model.

We categorized the internal state of an animal into active and resting. We based state classification on the predicted sequence of structural behaviors from the behavior model. We defined an animal in state active, when it primarily displays the behaviors browsing, walking, trotting, galloping and 'others' (in the following summarized as active behaviors because all involve physical motions) for a duration of at least $7 \mathrm{~min}$. We decided for a minimum duration to exclude short peaks of active behaviors (e.g., relocation, scratching) during resting states. Similarly, we consider an animal in state resting, when it displays primarily lying or standing behavior (in the following, summarized as passive behaviors) for a duration of at least $7 \mathrm{~min}$. We used standing and lying behavior because roe deer displayed both behaviors while resting (personal observations). Here, the minimum duration of 7 min compromises between roe deer that sometimes rest (e.g., lay down) between two browsing periods for short periods and deer that are highly alert (stand and scan the surrounding) for several minutes (see discussion). The length of $7 \mathrm{~min}$ was derived from discussions with several roe deer experts. We implemented these rules in the following way. First, we applied the behavior model to the acceleration data of interest. We merged the predicted behaviors lying and standing as passive behaviors and all other behaviors as active behaviors and calculated the proportion of active behavior per burst $\left(p_{\text {active }}\right)$. We then calculated a moving average with a window width of seven minutes over the time series $p_{\text {active }}$. The generated time series showed obvious periods with high activity and periods with low activity (a graphical illustration is provided in Additional file 4: Fig. S4). To find the transition points between active and resting states, we calculated the crossing points between the moving average and a threshold value. As selection criterion for the optimal threshold value, we calculated the total proportion of time in state active for a sequence of threshold values $(0,0.05,0.1, \ldots, 1)$ and plotted both. Due to the primarily 'passive' and thus very homogeneous character of resting states, we wanted to select a threshold value that (1) maintains low variation within resting states and (2) reliably discriminates resting and active states. We, therefore, argue for the smallest threshold value after the sharp decline in the proportion of time in state active that occurs, when resting states are separated 
from active states. The choice of the window size and the threshold value affected the starting/ending points of active states. To better match the exact starting/ending points, we scanned the time windows where the threshold value was intersected a second time and assigned the final starting/ending points of active states to the timestamp of the last/first burst at which its $p_{\text {active }}$ actually exceeds the threshold value. This procedure also detected active states that were shorter than $7 \mathrm{~min}$. According to our definition of active states, we finally removed these.

According to the common definition of vigilance behavior, we defined an animal as vigilant when it is standing still, with the head raised above shoulder height, while being in state active [15]. The final behavior model had difficulties to differentiate between the behaviors standing and lying. This resulted in many standing events being classified as false lying events. It is very unlikely that an animal lies down for short periods of time (e.g., a few seconds) while being in state active. Hence, all predicted lying events inside an active state are presumably true standing events. Based on this assumption, we merged both behaviors for vigilance detection and joined the corresponding splitting rules. Additionally, the behaviors standing and lying were solely classified based on parameters that reflect the dynamic body acceleration. For vigilance detection, we explicitly wanted to integrate the posture head up as a classification rule to separate out events where the animal is standing with the head in low or middle position. Hence, we constructed the vigilance model by manually combining the splitting rules that predicted the behaviors standing and lying from the behavior model, and the splitting rules that predicted the posture head up from the posture model. We predicted vigilance behavior inside all active states. To evaluate this approach, an experienced person assessed two video recordings per animal and tagged all occurrences of vigilance behavior according to expert judgment. After, we compared these with the predictions from the vigilance model and calculated accuracy, sensitivity and positive predictive value for each animal.

\section{Model application}

We predicted animal behavior, state and vigilance behavior for a two-year-old male roe deer (animal id 53) in March of 2013. To spatially and temporally plot vigilance levels, we projected the collected GPS-positions onto the time series of active and resting states and assigned each GPS-position to one of the two states. We then calculated the proportion of time spent vigilant in a time window of $10 \mathrm{~min}$ around each active GPS-position. We selected only active GPS-positions that were situated at least $15 \mathrm{~min}$ away from the transition points in order to prevent potential bias when the animal switches from a resting to active state and vice versa. Additionally, we plotted the detected active states over the time of day (actogram), calculated the proportion of time in state active during day vs night and time budgets for the predicted structural behavior categories.

\section{Results}

We documented all behavior categories of interest for eight animals in the field. Five additional animals were observed just for short periods of time with an incomplete set of behaviors and were therefore not considered for this analysis. In total, we behavior-tagged more than $15 \mathrm{~h}$ of acceleration data. The amounts of behaviortagged acceleration data differed substantially between the animals (Additional file 3: Fig. S3). Browsing and lying were observed most frequently.

The final classification model discriminated all behaviors of interest (Fig. 3; cross-validation plot: Additional file 5: Fig. S5 left). The model separated the behaviors standing and lying from the active behaviors by the mean dynamic body acceleration (mdba_xyz_w2). Browsing behavior was subsequently split by the corrected static body acceleration of the $x$ axis (sba_x_c) and, thus, by the posture of an animal. The behaviors walking, trotting and galloping were again discriminated by predictor variables that correspond to the dynamic body acceleration. Applied to the test dataset, the classification model correctly predicted all behaviors in $71 \%$ of the cases (range between animals 66-79\%). The confusion matrix (Table 4) revealed that most erroneous predictions were distributed in behavior categories that are characterized by similar body posture and dynamic body acceleration. Thus, the model mixed standing with lying, browsing with walking and trotting with galloping. Errors concerning the behavior 'others' were distributed over all behaviors. For most behavior categories, sensitivity and positive predictive value were comparable between animals (Fig. 4, left).

Optimal tree size of the posture model according to the one standard deviation rule was 12 (Additional file 5: Fig. S5 right). This and the next smaller model did not yield an obvious improvement to the model. We, therefore, pruned the tree to a size of three (Fig. 5). The model discriminated all three postures based on the corrected static body acceleration of the $x$ axis. The overall model accuracy for the posture model was $88 \%$ (range between animals $78-91 \%$ ). The posture model made most errors by mixing head down with head middle, and head middle with head up, but rarely mixed head up with head down (Table 5). Sensitivity and positive predictive value were comparably high for all animals and postures (Fig. 4, right).

According to the criteria for an optimal threshold value to discriminate between active and resting states, 


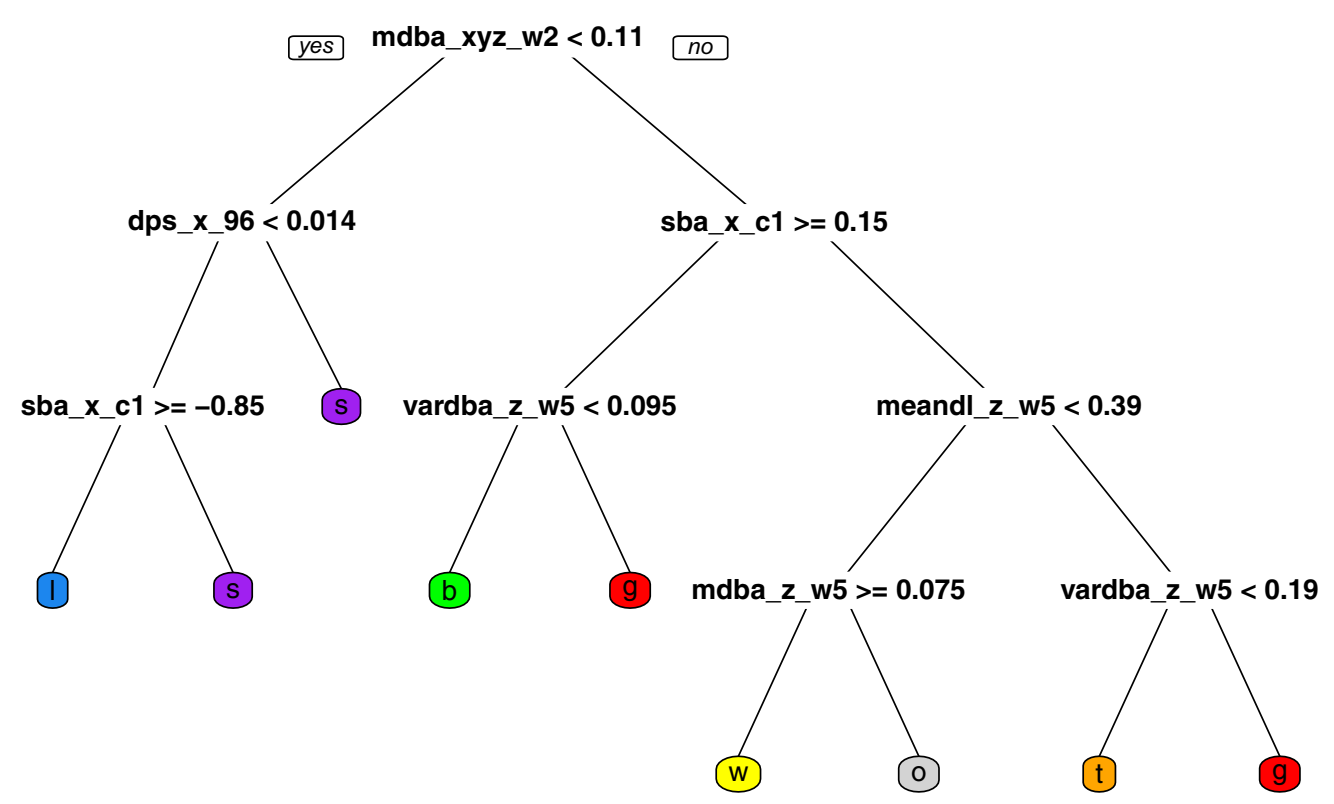

Fig. 3 Classification tree of the behavior model. The model classified behavior into lying $(h)$, standing $(s)$, browsing $(b)$, walking $(w)$, trotting $(t)$, galloping $(g)$ and 'others' $(0)$. See Table 3 for a detailed description of the predictor variables

Table 4 Confusion matrix of the behavior model

\begin{tabular}{|c|c|c|c|c|c|c|c|c|}
\hline & & \multicolumn{7}{|c|}{ Reference } \\
\hline & & Lying & Standing & Browsing & Walking & Trotting & Galloping & Others \\
\hline \multirow[t]{7}{*}{ Prediction } & Lying & 3545 & 1142 & 0 & 6 & 0 & 0 & 91 \\
\hline & Standing & 272 & 1811 & 103 & 71 & 0 & 3 & 297 \\
\hline & Browsing & 2 & 133 & 3450 & 469 & 11 & 4 & 191 \\
\hline & Walking & 11 & 354 & 310 & 2719 & 96 & 32 & 773 \\
\hline & Trotting & 0 & 9 & 0 & 102 & 974 & 86 & 29 \\
\hline & Galloping & 1 & 37 & 7 & 29 & 153 & 1317 & 126 \\
\hline & Others & 169 & 514 & 130 & 400 & 3 & 4 & 1287 \\
\hline
\end{tabular}

The confusion matrix divides all data points according to the reference ( $x$ axis, true behavior) and the behavior prediction from the classification model ( $y$ axis). The number of data points correctly classified is shown in italics

we selected a threshold value of 0.05 (Additional file 6: Fig. S6). Thus, when an animal displayed more than $5 \%$ active behaviors within a time window of $7 \mathrm{~min}$, it made the transition from resting to active and vice versa (with the exception that the resulting state lasted for at least $7 \mathrm{~min}$ ). We constructed our classification model for vigilance detection by combining the splitting rule that discriminated the behaviors standing and lying (mdba xyz_w2 $<0.11$, behavior model) with the splitting rule for posture head up (sba_x_c $<-0.4$, posture model). Compared to expert judgment of vigilance behavior (in total $230 \mathrm{~min}$ of video recordings evaluated), the model predicted vigilance behavior with a mean accuracy of $96.02 \%$ (range between animals 92.56-98.95\%), mean sensitivity of $98.35 \%(95.39-100 \%)$ and mean positive predictive value of $96.85 \%$ (91.61-98.97\%).

In March of 2013, animal 53 displayed 309 active states (Fig. 6, left) with a mean number of 10.3 active states per $24 \mathrm{~h}$ (sd 2.53). On average, the active states accounted for $42 \%$ of the day (sd 0.05 ). We observed a slight tendency to higher activity levels during nighttime on most days (Fig. 6, right). The mean duration of an active state was $59 \mathrm{~min}$ (sd 41). An average resting state lasted $81 \mathrm{~min}$ (sd $48 \mathrm{~min}$ ). According to the predicted behaviors from the behavior model, during active states, animal 53 spent most time browsing (62\%), followed by standing (16\%), lying (6\%), walking (7\%) and 'others' (6\%) (Additional file 7: Fig. S7). Trotting and galloping behavior was 


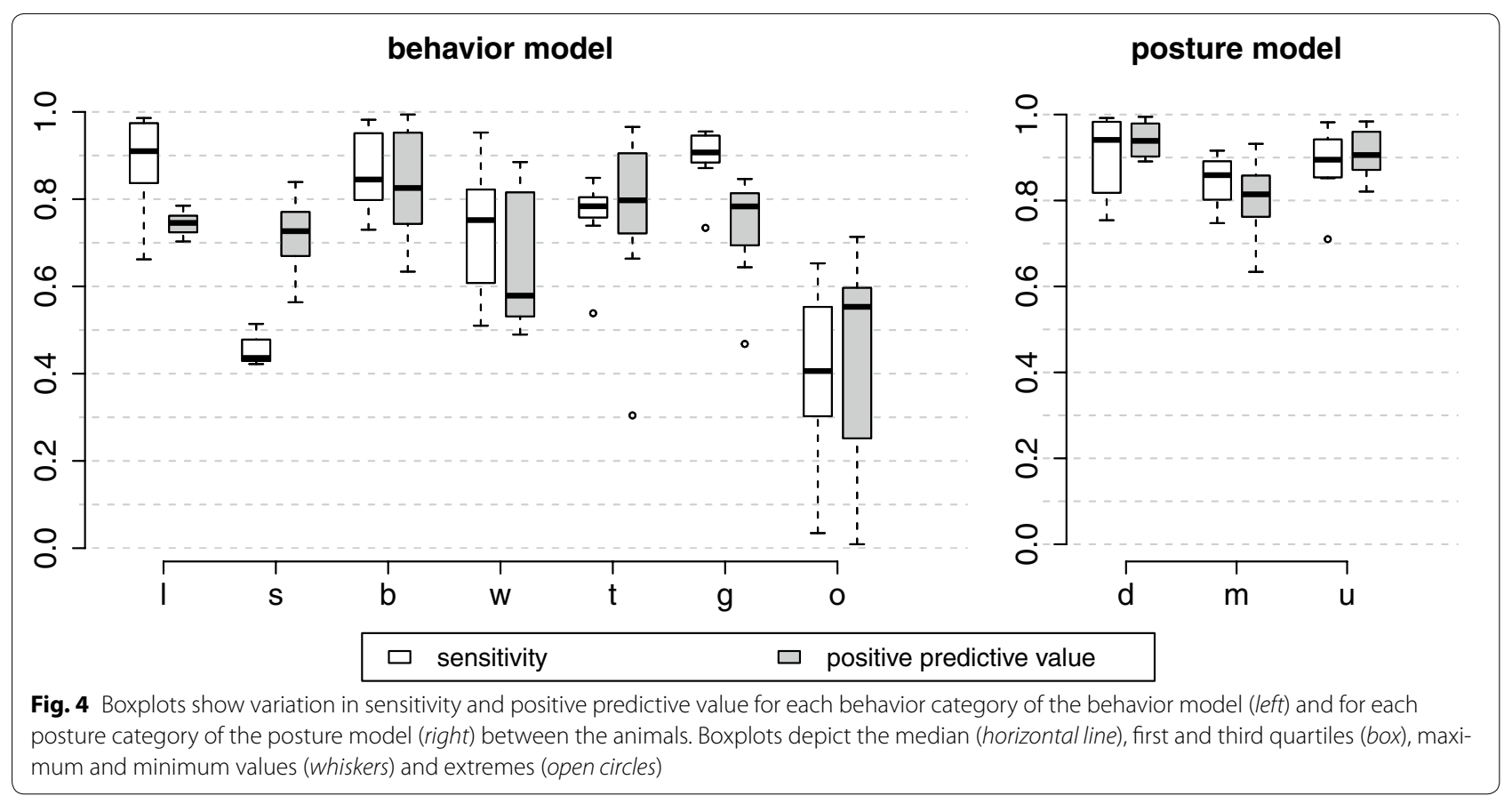

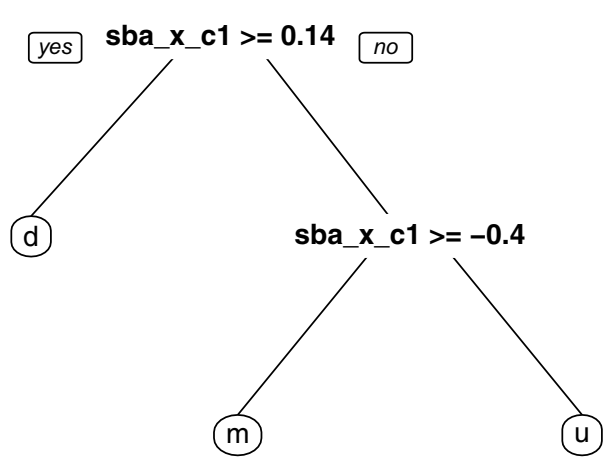

Fig. 5 Classification tree of the posture model. The model classified the posture of an animal's neck into down $(d)$, middle $(m)$ and up $(u)$. See Table 3 for a detailed description of the predictor variables

Table 5 Confusion matrix of the posture model

\begin{tabular}{lllll}
\hline & & \multicolumn{3}{l}{ Reference } \\
\cline { 3 - 5 } & & Down & Middle & Up \\
\hline Prediction & Down & 3615 & 225 & 16 \\
& Middle & 378 & 3214 & 447 \\
& Up & 7 & 350 & 3537 \\
\hline
\end{tabular}

The confusion matrix divides all data points according to the reference $(x$ axis, true posture) and the posture prediction from the classification model ( $y$ axis).

The number of data points correctly classified is shown in italics observed less than $1 \%$ of the time. During resting states, animal 53 was mostly lying (94\%) and standing (4\%). The spatial distribution of resting GPS-positions was clearly more clumped than that of active GPS-positions (Fig. 7, left). Animal 53 was, on average, vigilant for 8.5\% of the time during active states. Spatial differences in the observed vigilance behavior were not clearly visible (Fig. 7, right). However, when vigilance levels are plotted temporally (Fig. 8), it appears that animal 53 was more vigilant during daylight and reduced its vigilance level $2 \mathrm{~h}$ after sunset.

\section{Discussion}

The primary aims of this study were to develop and evaluate models for an automated classification of animal behavior, internal state and vigilance behavior based on raw acceleration data of Roe deer. We were able to successfully implement all three tasks.

The classification model for animal behavior discriminated all structural behaviors with an overall accuracy of $71 \%$. However, the model struggled with reliably discriminating between behaviors that share similar acceleration signatures. This was especially the case for the behaviors standing and lying, where $29 \%$ of the standing data points were erroneously classified as lying. In both, the animal is characterized by almost no body movement and an identical neck posture that makes differentiation 


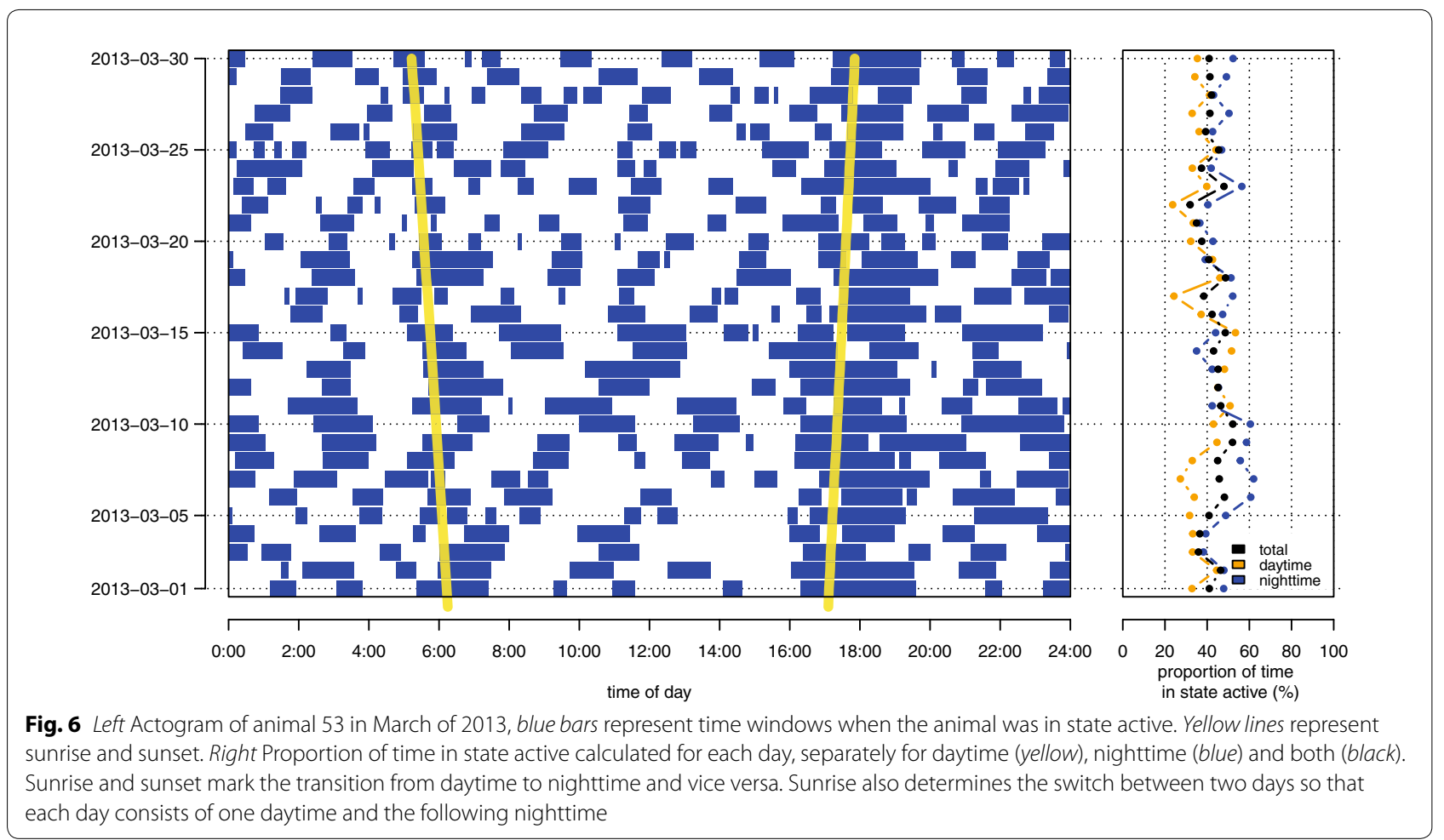

difficult. Posterior plausibility checks are an option to correct for presumably misclassified behaviors that are unlikely to occur in the specific context. We implemented this approach for vigilance detection and joined the classification rules that predicted lying and standing behavior due to unlikely occurrence of lying behavior while being classified as state active. In doing so, we could reduce false predictions during active states, but were not able to reduce them during resting states. Using internal states as contextual information for error correction could be beneficial for many species whenever internal states are featuring the presence or absence of specific behaviors. Visual inspections of model predictions also revealed that false predictions often affected only a few data points that were surrounded by true predictions. This could provide further options for posterior model improvement by implementing moving windows that vote for the most frequent behavior inside the window. Detailed evaluation of model performance is especially important, if the model is to be extrapolated to other animals or outside the observation period. Variations in the acceleration data between and within animals might be caused by varying sensor measurement, collar tightness, collar position, individual-specific variations in behaviors or changing environmental conditions (e.g., seasonal changes in vegetation height or changing habitat availability). Shamoun-Baranes et al. [7] therefore suggested to develop individual-based models, which is most often technically unfeasible, especially in terms of timeconsuming field observations and elusive study species. Instead, we aimed at constructing one population-based model and controlled potential bias by implementing collar-specific parameter correction (parameter sba_x_c), homogenization of the training dataset and cross-validation based on the animals. Additionally, we calculated animal-specific performance parameters for each behavior category. These were in similar ranges, indicating that overfitting toward individual animals was hardly present. Only for a few animals we observed varying values for the behaviors trotting and galloping, which were most likely caused by the low number of data points. The behavior category 'others' was less predictable. Here, we pooled several behaviors despite their divergent acceleration signatures. These probably interfered with the acceleration signatures of the main behavior categories. Once one of these behaviors is targeted, we recommend to split 'others' into more homogeneous behavior categories. Finally, the choice of a rather simple modeling technique allowed for an easy evaluation of the classification rules. We identified one split, in particular, that needs special attention 

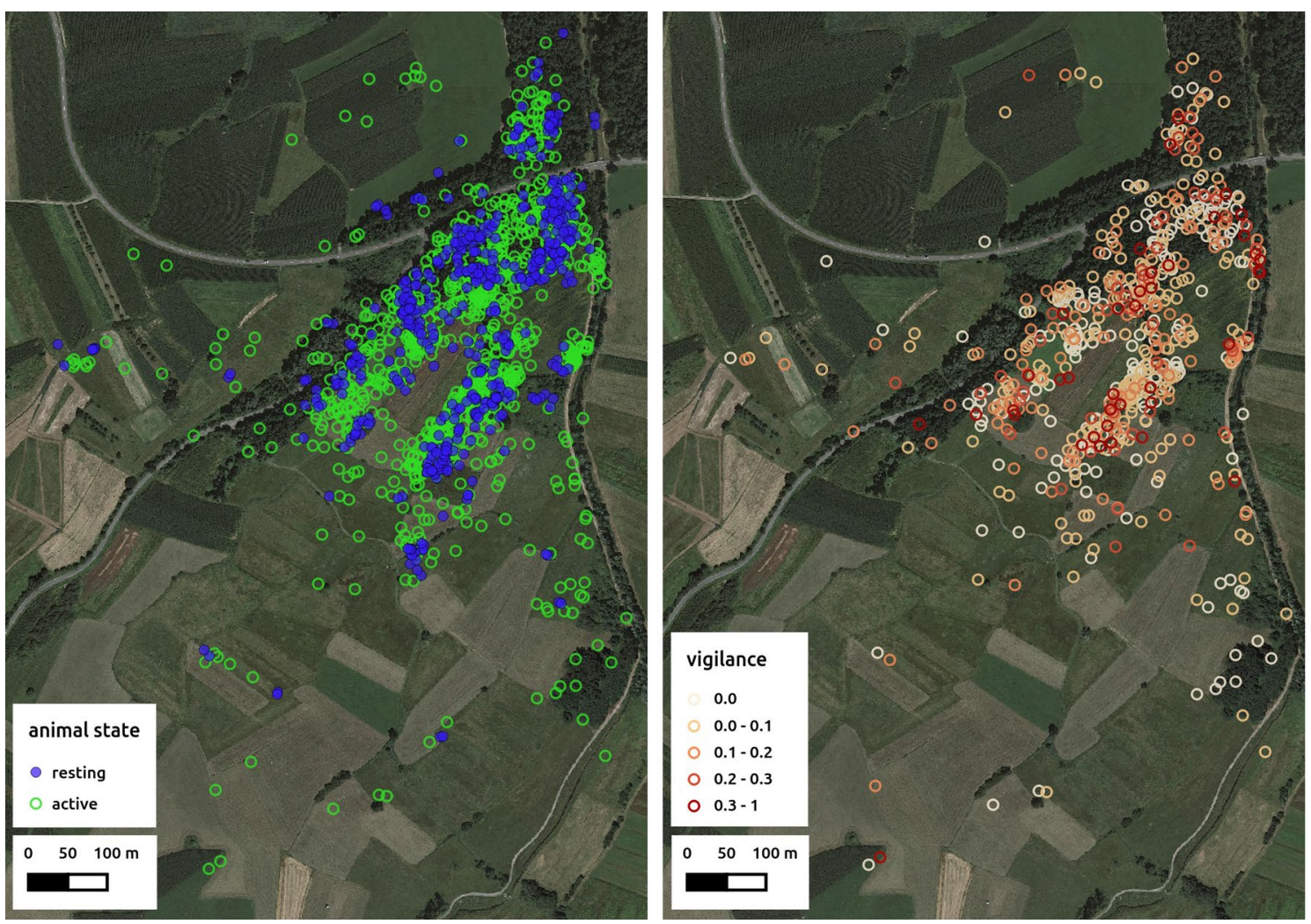

Fig. 7 Animal locations categorized according to resting and active states (left), and active locations categorized according to different vigilance levels (right) for animal 53 in March of 2013. A GPS-location is marked as an active/resting location when it was collected during an active/resting period of animal 53. Vigilance is calculated as the proportion of time spent vigilant during active states, calculated in a time window of 10 min around each active GPS-location

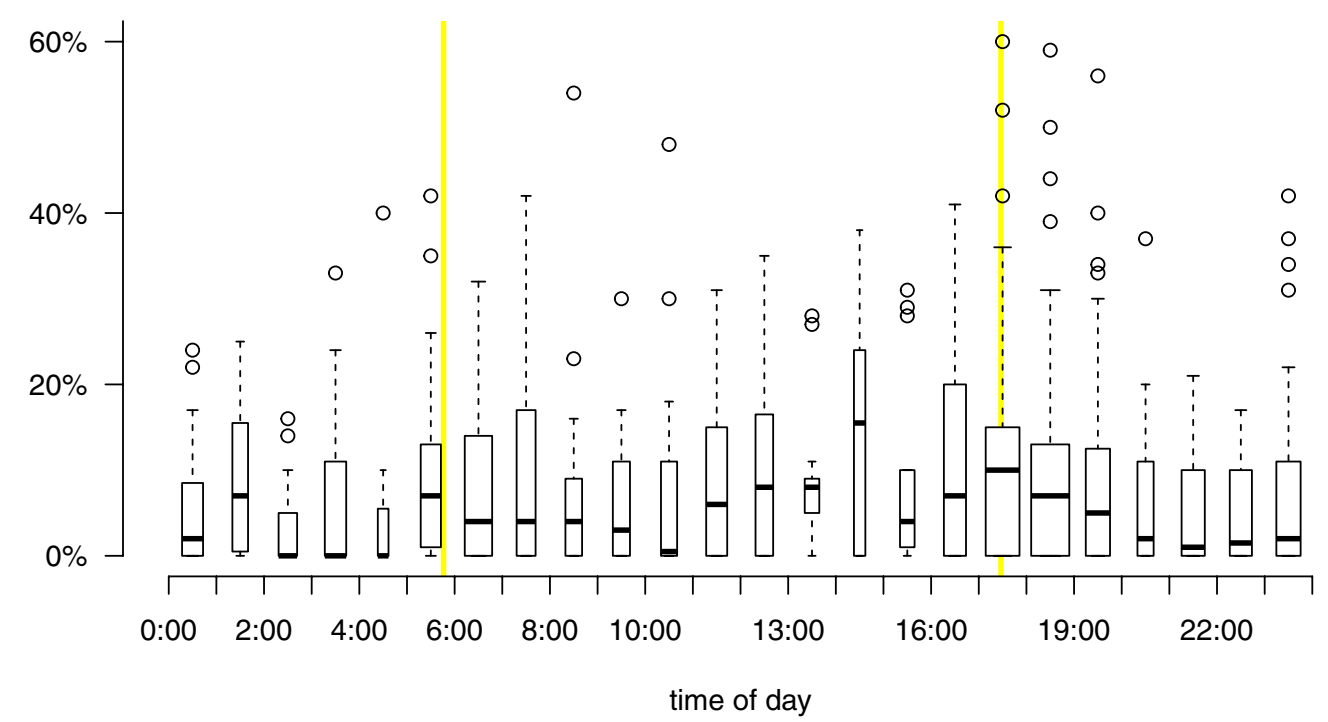

Fig. 8 Boxplot of the predicted proportion of time spent vigilant while being in state active, separated for each hour of the day, for animal 53 in March of 2013. Yellow lines represent sunrise and sunset. Box widths are adjusted proportionally to the number of data points 
when model prediction for other seasons is intended. An animal is classified as browsing when the dynamic body acceleration is in a specific range (odba_xyz_w2 $<0.11$ and var_dba_z_w5 $<0.1$ ) and, more importantly, when the head moves down (sba_x_c $\geq 0.15$ ). This specific splitting rule might have been influenced by our observation period during the winter months when the vegetation is normally low. During the growing season or in environments that are characterized by high vegetation, animals might not be forced to lower their heads to ingest food. This would produce false predictions of category walking or 'others' under the current model. Unfortunately, we were not able to do behavioral observations during the growing season or in other habitat types due to the low visibility of the animals. Thus, when model extrapolation is intended, effective evaluation of model predictions is required and could be further supported by seasonal or habitat-specific histograms of the predictor variables to check for data consistency (see Kroeschel in preparation).

In comparison with previous studies, which established the predictor variables based on the acceleration burst or segments of fixed width, we modified the classification procedure by calculating the predictor variables for each data point with moving windows of varying widths. We did not aim at comparing this approach with an approach using fixed segments. However, we expect that using each data point was advantageous because we generated an extended set of predictor variables that allowed for finer adaption to behavior-specific acceleration signatures. Further, we delegated the choice of the optimal width for each behavior to the classification model. We were also able to reliably detect changing behaviors inside a burst. However, parameters that capture features of the dynamic body acceleration within a moving window were still prone to cause false predictions at the transition points of two behaviors. This was apparent when the model would sometimes predict short intervals (usually less than one second) as false moving or false 'others' behavior when animals switched from 'standing with head up' to 'browsing with head down.' But even in these cases, the true change of behavior was usually detected very quickly, allowing for easy post-processing of the false prediction (see "Discussion" section above).

Discriminating behavior into structural behavior categories is usually straightforward because it involves clearly observable behavior categories. In state detection, this is often not the case, as we are not yet able to monitor an animal's mind directly. Nevertheless, states can be discriminated, particularly, when they differentiate in the value range of observable and, in case of automated classification, measurable parameters. We differentiated states based on expert-based rules that were applied to a sequence of structural behaviors. After exploratory comparison of model predictions and video recordings, we are confident that model predictions coincided with expert judgment in most circumstances. However, the model will fail when animals are either in state active but mainly vigilant for more than 7 min (results in false resting and thus missed vigilance) or lying down for less than $7 \mathrm{~min}$ between active states (results in false active and false vigilance). Adjusting either the width of the time window or the threshold value would not substantially improve the model because false model predictions simply shift to one or the other case. In these situations, human observers are superior because they are able to acquire additional information (e.g., posture of ears, body tension, external stimuli) that facilitate correct discrimination. Unfortunately, we lacked this information with our sensors, although techniques like measurements of micromovements [29] or heart beat [30] promise to fill this gap soon. Additionally, animals sometimes gradually transition from one state to another or display active behaviors (e.g., grooming) after lying down. These intermediate states, which mainly occur at the transition points, poorly fit into a binary discrimination in active and resting and can only be overcome by a finer discrimination of states. Not only the proportion but also the temporal sequence of specific structural behaviors promises to hold important information for discriminating these more complex states. The wide array of techniques being developed for state detection in movement ecology [4] might provide helpful tools to process this extended set of parameters.

In recent years, the concept of internal states gained increasing recognition in behavioral ecology, because it allows for the integration of alternating needs into statistical models [13, 31]. So far, internal states were always deduced from movement pattern [2-4], physiological measurements [32] or activity measurements [11, 33, 34]. Combining these with behavioral parameters could substantially improve state detection, because fine-resolution behavioral data facilitate plausible interpretation and, thereby, detailed discrimination of states.

Very high agreement with expert judgment of vigilance behavior confirmed that even complex behaviors can be predicted with acceleration data. Moreover, the observed vigilance levels were comparable to previous field studies with roe deer ([35]: median vigilance $10.5 \%$, range $<0.01-$ 72.7\% (Poland); [20]: mean 24.21\%, sd 14.55\% (France), both sampled outside the hunting period). In contrast to these, we were able to continuously monitor vigilance 
behavior in focal animals, even when the animal was hidden in cover, and without introducing any disturbance caused by observer presence in the field. However, when applying the presented approach, a few points have to be considered. The transition points between active and resting states are especially prone to misclassification errors when resting animals are erroneously classified as active. To prevent bias, we omitted the transition points by excluding a buffer of $10 \mathrm{~min}$ around these and, thus, focused on the core active states. Unfortunately, we were not able to control for false predictions of vigilance behavior when animals were lying down for short periods of time. Here, we currently recommend being cautious about sampling intervals of very high vigilance due to the risk of misclassified lying behavior. Many researchers focused field observations of vigilance on animals that were foraging. We differentiated active and resting states and excluded resting states for monitoring of vigilance. Thus, we relaxed strict foraging periods and also allowed for periods when the animal was, e.g., primarily moving. We do think that this simplification was adequate because (1) active states of roe deer during wintertime are mainly dedicated to gaining energy (which was indicated by high proportion of browsing behavior in animal 53 during those states) and (2) other active behaviors besides foraging (e.g., moving) are likewise characterized by limited predator detection probabilities [36] and, thus, should be traded with vigilance behavior to maintain safety. State classification, nevertheless, can be easily modified by targeting structural behaviors that are distinctive for more specific states (e.g., state foraging can be discriminated by a minimum proportion of browsing behavior inside the moving window).

We pioneered this approach with data from roe deer, but each step of the approach can be easily adapted to other large herbivores. Other species, however, require separate behavioral observations to train classification models and species-specific rules to discriminate internal states.

\section{Conclusion}

By predicting behavior, state and vigilance to an exemplary dataset, we demonstrated diverse applications and the huge potential behind acceleration data that can be assembled with the presented approach. In particular, continuous monitoring of vigilance behavior, in combination with detailed behavior budgeting and spatiotemporal allocation of both, will provide new insights in how large herbivores perceive and control predation risk [14, 17], will allow to test scientific hypotheses and models [e.g., 37] and, additionally, will provide helpful tools for wildlife management [e.g., 38].

\section{Additional files}

Additional file 1: Fig. S1. Picture of a collared roe deer with schematic representation of the acceleration axes. $X$ axis measures acceleration in forward-backward horizontal motion (surge), y axis measures left-right horizontal motion (sway), and $z$ axis measures up-down vertical motion (heave).

Additional file 2: Fig. S2. The graph shows the density distribution of the static body acceleration of the $x$ axis (sba_ $x$, black line) from animal 15. All animal-specific density distributions of the sba_x values showed two distinct peaks that correspond to an animal's most frequent postures 'head up' (1) and 'head down' (2). The posture 'head up' and, thus, the position of the first peak should be similar for all animals. However, we observed differences between the animals (see red line that shows the mean of the first peak from all collared animals) which were very likely caused by variating offset values (Kroeschel in preparation). We therefore calculated a corrected static body acceleration of the $x$ axis (sba_x_c) by subtraction a collar-specific correction value (a) from the sba_x values and, thus, centered the first maximum (posture head up 1) for all animals (see also Table 3).

Additional file 3: Fig. S3. Total number of behavior-tagged data points per animal and behavior class (left), selected datasets for model training (middle) and for model testing (right).

Additional file 4: Fig. S4. Proportion of active behavior per burst ( $p_{\text {active }}$ blue bars) over time of the day for animal 53 on March 1, 2013. The red line shows a moving average with a window width of $7 \mathrm{~min}$. For state detection, the crossing points between the moving average and the threshold value (dotted horizontal line) were calculated, adjusted to the actual starting/ending positions of the active states and, finally, checked for a minimum duration of $7 \mathrm{~min}$. The bold black lines show the final active states. The points below show the point in time when the collar took a GPS-position. Blue points indicate GPS-positions that were taken during resting states; green points indicate GPS-positions that were taken during active states. Framed green points were selected as reference points for the calculation of vigilance levels.

Additional file 5: Fig. S5. Cross-validation plot of the behavior model (left) and cross-validation plot of the posture model (right). The red lines mark the positions of model pruning.

Additional file 6: Fig. S6. Total proportion of time in state active calculated for a sequence of threshold values. The red circle marks the selected threshold value.

Additional file 7: Fig. S7. Behavior time budgets for animal 53 predicted by the behavior model in total (left), during resting states (middle) and active states (right).

Additional file 8. R-Script 01_datapreparation.R.

Additional file 9. R-Script 02-01_analysis_behavior_model.R.

Additional file 10. R-Script 02-02_analysis_posture_model.R.

Additional file 11. R-Script 03-01_model_application_datapreparation.R.

Additional file 12. R-Script 03-02_model_application_exploratory.R.

Additional file 13. R-Script 99_f_error-structure.R.

Additional file 14. R-Script 99_f_predict_behavior_vigilance.R.

Additional file 15. R-Script 99___predict_state.R.

\section{Authors' contributions}

MK designed the study, conducted the data analysis and wrote the manuscript. Fieldwork and behavior-tagging of acceleration data was done by FWe, FWi and MK. IS and BR contributed to analysis, theory and writing the manuscript. All authors read and approved the final manuscript. 


\begin{abstract}
Author details
${ }^{1}$ Chair of Wildlife Ecology and Wild life Management, University of Freiburg, Tennenbacherstr. 4, 79106 Freiburg, Germany. ${ }^{2}$ Forest Research Institute of Baden-Wuerttemberg, Wonnhaldestrasse 4, 79100 Freiburg, Germany. ${ }^{3}$ Irstea, UR EMGR, Université Grenoble Alpes, 2 rue de la Papeterie, BP 76, 38402 St-Martin-d'Hères, France. ${ }^{4}$ Biogeographical Modelling, Bayreuth Center of Ecology and Environmental Research BayCEER, University of Bayreuth, Universitätsstraße 30, 95440 Bayreuth, Germany.
\end{abstract}

\section{Acknowledgements}

First of all, we thank the German Academic Scholarship Foundation (Studienstiftung des Deutschen Volkes) for supporting MK with a Ph.D. scholarship. We also thank Noldus Information Technology for providing their software 'Observer XT.'We are grateful to all people that assisted in the field. Finally, we thank Stephanie Witczak, Benedikt Gehr, Lino Kämmerle, Luca Corlatti and two anonymous reviewers for their insightful and constructive comments.

\section{Competing interests}

The authors declare that they have no competing interests.

\section{Availability of data and materials}

All R-Scripts are provided in the additional files (Additional files 8, 9, 10, 11, 12, 13, 14, 15). Data are available upon request.

\section{Ethics approval and consent to participate}

All capture, tagging and monitoring protocols were approved by the animal welfare and hunting administration of Baden-Wuerttemberg, Germany.

\section{Funding}

Roe deer telemetry was funded by the Federal State of Baden-Wuerttemberg (Landesjagdabgabe). MK was financed by a Ph.D. scholarship of the German Academic Scholarship Foundation (Studienstiftung des deutschen Volkes).

\section{Publisher's Note}

Springer Nature remains neutral with regard to jurisdictional claims in published maps and institutional affiliations.

Received: 31 May 2016 Accepted: 17 March 2017

Published online: 04 May 2017

\section{References}

1. Cagnacci F, Boitani L, Powell RA, Boyce MS. Animal ecology meets GPSbased radiotelemetry: a perfect storm of opportunities and challenges. Philos Trans R Soc Lond B Biol Sci. 2010;365:2157-62.

2. Merrill E, Sand H, Zimmermann B, McPhee H, Webb N, Hebblewhite M, et al. Building a mechanistic understanding of predation with GPS-based movement data. Philos Trans R Soc Lond B Biol Sci. 2010;365:2279-88.

3. Nams VO. Combining animal movements and behavioural data to detect behavioural states. Ecol. Lett. 2014;17:1228-37.

4. Gurarie E, Bracis C, Delgado M, Meckley TD, Kojola I, Wagner CM. What is the animal doing? Tools for exploring behavioural structure in animal movements. J Anim Ecol. 2016;85:69-84

5. Brown DD, Kays R, Wikelski M, Wilson R, Klimley A. Observing the unwatchable through acceleration logging of animal behavior. Anim Biotelem. 2013;1:20.

6. Shepard E, Wilson RP, Quintana F, Gómez Laich A, Liebsch N, Albareda D, et al. Identification of animal movement patterns using tri-axial accelerometry. Endanger Species Res. 2008;10:47-60.

7. Shamoun-Baranes J, Bom R, van Loon EE, Ens BJ, Oosterbeek K, Bouten W. From sensor data to animal behaviour: an oystercatcher example. PLoS One. 2012;7:e37997. doi:10.1371/journal.pone.0037997.

8. Nathan R, Spiegel O, Fortmann-Roe S, Harel R, Wikelski M, Getz WM. Using tri-axial acceleration data to identify behavioral modes of free-ranging animals: general concepts and tools illustrated for griffon vultures. J Exp Biol. 2012;215:986-96.

9. McClune DW, Marks NJ, Wilson RP, Houghton JD, Montgomery IW, McGowan NE, et al. Tri-axial accelerometers quantify behaviour in the
Eurasian badger (Meles meles): towards an automated interpretation of field data. Anim Biotelem. 2014;2:5.

10. Sakamoto KQ, Sato K, Ishizuka M, Watanuki Y, Takahashi A, Daunt F, et al. Can ethograms be automatically generated using body acceleration data from free-ranging birds? PLoS One. 2009;4:e5379. doi:10.1371/journal. pone.0005379.

11. Löttker P, Rummel A, Traube M, Stache A, Šustr P, Müller J, et al. New possibilities of observing animal behaviour from a distance using activity sensors in GPS-collars: an attempt to calibrate remotely collected activity data with direct behavioural observations in red deer Cervus elaphus. Wildl Biol. 2009;15:425-34.

12. Martin P, Bateson P. Measuring behaviour an introductory guide. Cambridge: Cambridge University Press; 2007.

13. Nathan R, Getz WM, Revilla E, Holyoak M, Kadmon R, Saltz D, et al. A movement ecology paradigm for unifying organismal movement research. Proc Natl Acad Sci USA. 2008;105:19052-9.

14. Lima SL, Dill LM. Behavioral decisions made under the risk of predation: a review and prospectus. Can J Zool. 1990;68:619-40.

15. Caro T, Girling S. Antipredator defenses in birds and mammals (interspecific interactions). Chicago: University of Chicago Press; 2005.

16. Beauchamp G. Animal vigilance: monitoring predators and competitors. London: Academic Press; 2015.

17. Brown JS, Laundré JW, Gurung M, Laundre JW. The ecology of fear: optimal foraging, game theory, and trophic interactions. J Mammal. 1999;80:385.

18. Fortin D, Boyce MS, Merrill EH, Fryxell JM. Foraging costs of vigilance in large mammalian herbivores. Oikos. 2004;107:172-80.

19. Childress MJ, Lung MA. Predation risk, gender and the group size effect: does elk vigilance depend upon the behaviour of conspecifics? Anim Behav. 2003;66:389-98.

20. Benhaiem S, Delon M, Lourtet B, Cargnelutti B, Aulagnier S, Hewison AJM, et al. Hunting increases vigilance levels in roe deer and modifies feeding site selection. Anim Behav. 2008;76:611-8.

21. Brown DD, LaPoint S, Kays R, Heidrich W, Kümmeth F, Wikelski M. Accelerometer-informed GPS telemetry: reducing the trade-off between resolution and longevity. Wildl Soc Bull. 2012;36:139-46.

22. Kuemmeth F, Heidrich W. e-obs GPS-acceleration-tags application note: how to use the acceleration sensor, interpret, analyse its data and how to get values in m/s2. e-obs. 2011.

23. Wilson RP, Shepard E, Liebsch N. Prying into the intimate details of animal lives: use of a daily diary on animals. Endanger Species Res. 2008:4:123-37.

24. Watanabe S, Izawa M, Kato A, Ropert-Coudert Y, Naito Y. A new technique for monitoring the detailed behaviour of terrestrial animals: a case study with the domestic cat. Appl Anim Behav Sci. 2005;94:117-31.

25. Shepard E, Wilson RP, Halsey L, Quintana F, Gómez Laich A, Gleiss A, et al. Derivation of body motion via appropriate smoothing of acceleration data. Aquat Biol. 2008;4:235-41.

26. Banerjee D, Biswas S, Daigle C, Siegford JM. Remote activity classification of Hens using wireless body mounted sensors. In 2012 Ninth international conference on wearable and implantable body sensor networks. IEEE. 2012;107-12.

27. Therneau T, Atkinson B, Ripley B. rpart: recursive partitioning and regression trees. R package version 3.1; 2015. https://CRAN.R-project.org/ package $=$ rpart.

28. R Core Team. R: a language and environment for statistical computing. Vienna: R Foundation for Statistical Computing; 2015.

29. Wilson RP, Grundy E, Massy R, Soltis J, Tysse B, Holton M, et al. Wild state secrets: ultra-sensitive measurement of micro-movement can reveal internal processes in animals. Front Ecol Environ. 2014;12:582-7.

30. Signer C, RufT, Schober F, Fluch G, Paumann T, Arnold W. A versatile telemetry system for continuous measurement of heart rate, body temperature and locomotor activity in free-ranging ruminants. Methods Ecol Evol. 2010;1:75-85.

31. Roever CL, Beyer HL, Chase MJ, van Aarde RJ. The pitfalls of ignoring behaviour when quantifying habitat selection. Divers Distrib. 2013;20(3):322-33. doi:10.1111/ddi.12164

32. Jachowski DS, Singh NJ. Toward a mechanistic understanding of animal migration: incorporating physiological measurements in the study of animal movement. Conserv Physiol. 2015;3:cov035. 
33. Gottardi E, Tua F, Cargnelutti B, Maublanc M-L, Angibault J-M, Said $\mathrm{S}$, et al. Use of GPS activity sensors to measure active and inactive behaviours of European roe deer (Capreolus capreolus). Mammalia. 2010;74:355-62.

34. Gervasi V, Brunberg S, Swenson JE. An individual-based method to measure animal activity levels: a test on brown bears. Wildl Soc Bull. 2006;34:1314-9.

35. Sönnichsen L, Bokje M, Marchal J, Hofer H, Jędrzejewska B, Kramer-Schadt $\mathrm{S}$, et al. Behavioural responses of European roe deer to temporal variation in predation risk. Ethology. 2013;119:233-43.
36. Lima SL, Bednekoff PA. Back to the basics of antipredatory vigilance: can nonvigilant animals detect attack? Anim Behav. 1999;58:537-43.

37. Lima SL, Bednekoff PA. Temporal variation in danger drives antipredator behavior: the predation risk allocation hypothesis. Am Nat. 1999;153:649-59.

38. Grignolio S, Merli E, Bongi P, Ciuti S, Apollonio M. Effects of hunting with hounds on a non-target species living on the edge of a protected area. Biol Conserv. 2011;144:641-9.

\section{Submit your next manuscript to BioMed Central and we will help you at every step:}

- We accept pre-submission inquiries

- Our selector tool helps you to find the most relevant journal

- We provide round the clock customer support

- Convenient online submission

- Thorough peer review

- Inclusion in PubMed and all major indexing services

- Maximum visibility for your research

Submit your manuscript at

www.biomedcentral com/submit 